\title{
Alignment of Quasar Polarizations on Large Scales Explained by Warped Cosmic Strings. PART II: The Second Order Contribution
}

\author{
Reinoud Jan Slagter ${ }^{1,2}$ \\ ${ }^{1}$ Asfyon, Bussum, The Netherlands \\ ${ }^{2}$ Amsterdam University, Amsterdam, The Netherlands \\ Email: info@asfyon.com
}

How to cite this paper: Slagter, R.J. (2017) Alignment of Quasar Polarizations on Large Scales Explained by Warped Cosmic Strings. PART II: The Second Order Contribution. Journal of Modern Physics, 8, 163-180.

https://doi.org/10.4236/jmp.2017.82015

Received: January 17, 2017

Accepted: February 14, 2017

Published: February 17, 2017

Copyright $\odot 2017$ by author and Scientific Research Publishing Inc. This work is licensed under the Creative Commons Attribution International License (CC BY 4.0).

http://creativecommons.org/licenses/by/4.0/

\begin{abstract}
We find an azimuthal-angle dependent approximate wave like solution to second order on a warped five-dimensional manifold with a self-gravitating $U(1)$ scalar gauge field (cosmic string) on the brane using the multiple-scale method. The spectrum of the several orders of approximation show maxima of the energy distribution dependent on the azimuthal-angle and the winding numbers $n$ of the subsequent orders of scalar field. This breakup of the quantized flux quanta does not lead to instability of the asymptotic wavelike solution, due to the suppression of the n-dependency in the energy momentum tensor components by the warp factor. This effect is triggered by the contribution of the five dimensional Weyl tensor on the brane. This contribution can be understood as dark energy and can trigger the self-acceleration of the universe without the need of a cosmological constant. There is a striking relation between the symmetry breaking of the Higgs field described by the winding number and the $\mathrm{SO}(2)$ breaking of the axially symmetric configuration into a discrete subgroup of rotations about $180^{\circ}$. The discrete sequence of non-axially symmetric deviations, cancelled by the emission of gravitational waves in order to restore the $\mathrm{SO}(2)$ symmetry, triggers the pressure $T_{z z}$ for discrete values of the azimuthal-angle. There can be a possible relation between the recently discovered angle-preferences of polarization axes of quasars on large scales and our theoretical predicted angle-dependency and can be an evidence for the existence of cosmic strings. The discovery of the increase of polarization rate in smaller subgroups of the several largequasar groups (LQGs), the red shift dependency and the relative orientation of the spin axes with respect to the major axes of their host LQGs, point at a fractional azimuthal structure, were also found in our cosmic string model. This peculiar discontinuous large scale structure, i.e., polarizations directions of multiples of, for example, $\frac{\pi}{2}$ or $\frac{\pi}{4}$, can be explained by the spectrum of
\end{abstract}


azimuthal-angle dependent wavelike modes without the need of conventional density perturbations in standard 4D cosmological models. Carefully comparison of the spectrum of extremal values of the first and second order $\varphi$ dependency and the distribution of the alignment of the quasar polarizations is necessary. This can be accomplished when more observational data become available.

\section{Keywords}

Quasar Polarization, Cosmic Strings, Warped Brane World Models, $U(1)$ Scalar-Gauge Field, Multiple-Scale Analysis

\section{Introduction}

General relativity theory (GRT) is by far the most successful theory constructed by theoretical physicists. Its predictive power is impressive. Famous empirical confirmed examples are the Kerr black hole and the emission of gravitational waves by merging black holes. There are, however, predicted phenomena not yet detected by observations. An example is the axially symmetric (spinning) compact object, i.e., the Papapetrou or Lewis-van Stockum solution. Another well studied object is the self-gravitating cosmic string solution. For an overview, see the work of Vilenkin and Shellard [1] and Anderson [2]. Cosmic strings are $U(1)$ scalar gauge vortex solutions in general relativity in the framework of GUT's. This $U(1)$ scalar gauge field with a "Mexican hat" potential has lived up its reputation in the theory of superconductivity, where vortex lines occur as topological defects and in the standard model of particle physics. In cosmology it could trigger the inflationary period of expansion and could solve the horizon and flatness problem. It came as a surprise that the relativistic string-like vortex solution of Nielsen and Olesen [3] can be found in GRT [4] [5]. However, general relativistic cosmic string is still not found directly or indirectly by observations. The interest in cosmic strings faded away when one found inconsistencies with the power spectrum of the CMB: Cosmic strings cannot provide satisfactory explanation for the magnitude of the initial density perturbations from which galaxies and clusters grew. It turns out that the upper bound of the mass per unit length $G \mu \sim 10^{-6}$. Further, the special pattern of a lensing effect of cosmic strings is not found yet. Studies of the radiative effects of cosmic strings embedded in a FLRW spacetime show that cylindrical gravitational radiation is rapidly damped and is negligible in any physical regime [6]. These string-cosmology spacetimes essentially look like a scaled version of a string in a vacuum spacetime. There is, however, another possibility to test the existence of cosmic strings. The recently discovered alignment of quasar polarizations on very large scales [7] [8] could be explained by considering cosmic strings on a warped brane world spacetime [9] [10]. It was realized recently that cosmic strings could be produced within the framework of superstring theory inspired cosmological models and a revival of cosmic strings occurred. These so-called 
cosmic superstrings can play the role of cosmic strings in the framework of string theory or $M$-theory, i.e., brane world models. Supersymmetric GUT's can even demand the existence of cosmic strings. Super-massive strings with an energy density of $G \mu \gg 1$ are interesting [11] because their gravitational impact will be much stronger than GUT strings. They could be produced when the universe underwent phase transitions at energies much higher than the GUT scale. Brane world cosmological models were first proposed by Arkani-Hamed, Dimopoulos and Dvali (ADD) [12] [13] and Antoniadis et al. [14] and extended by Randall and Sundrum (RS) [15] [16]. In these models, the extra dimension can be very large compared to the ones predicted in string theory, i.e., of order of millimeters. The difference with the standard superstring model is that the compactification rely on the curvature of the bulk. The huge discrepancy between the electro-weak scale, $M_{\mathrm{EW}}=10^{3} \mathrm{GeV}$ and the gravitational mass scale, $M_{\mathrm{Pl}}=10^{19} \mathrm{GeV}$ (hierarchy problem) will be suppressed by the volume of the extra dimension, or the curvature in that region. This effect can also be achieved in the RS models by a warp factor. The weakness of gravity in these models are fundamental and the Planck energy could be of $\mathcal{O}(\mathrm{TEV})$ that may be accessible by LHC. It is possible that effective $4 \mathrm{D}$ Kaluza-Klein (KK)-modes are obtained from the perturbative 5D graviton. These KK-modes will be massive from the brane viewpoint. Further, one usually considers a fine-tuning between the tension on the brane ( $4 \mathrm{D}$ cosmological constant) and the $5 \mathrm{D}$ tension in order to ensure a zero effective cosmological constant. For an overview of brane world models, see, for example, Roy [17] [18] [19] and Shiromizu et al. [20] and references therein. Cosmic strings could have tremendous mass in the bulk, while their warped manifestations in the brane show consistency with the observed bound of $G \mu \sim 10^{-6}$ by the warp factor [21], even if its value was at the Planck scale. Wavelike disturbances triggered by the huge mass of the cosmic string in the bulk, could have indeed observational effects in the brane. Evidence of these objects would give us information at very high energies in the early stages of the universe. Maybe they might actually provide us the best observational window upon fundamental string theory. One reason for this conjecture is the possibility of the extension of fundamental strings into the bulk in warped spacetimes.

In this research we extend our previous work on the relation of the alignment of quasar polarizations with warped cosmic strings [10]. Recent research in this field [22] shows peculiar correlation of quasar polarization orientations in LQGs. The alignments can be parallel or perpendicular to each other. Ever other discretizations are not excluded. We shall see that this behaviour fits in our brane world model. However, there are some peculiar issues to be addressed. A network of long cosmic strings can be characterised by a single scaling length, the persistence length of the inter-string distance. Numerical simulations have shown that the evolution of the network must be scale-invariant. It is a great challenge to prove that there is a comparable scale-invariance in the alignments of the polarization vectors of quasars. By the discovery of high-redshift supermassive black holes, $z>6$, one could extend the investigation on quasar 
alignment to this era. There is already a tiny indication of $z$-dependency in the observed quasar polarization orientations [7]. Further, the formation of smallscale closed loops of cosmic strings can disturb the energy balance of long strings. In our model this problem is overcome by the warp factor: it makes the cosmic string more massive during the evolution.

In Section 2 we outline the multiple-scale method on a warped brane world spacetime. In Section 3 we calculate the metric perturbations to second order. In Section 4 we derive the matter field equations to second order and indicate the possible relation with axially symmetric instabilities caused by radiationreactions. In Section 5 we discuss the possible connection of the warp factor with conformal invariance. In the appendices we collected all the relevant equations in order to keep the main text readable and self-contained.

\section{The Multiple-Scale Approximation on a Warped Brane World Spacetime}

We will investigate vortex-like solution on a warped five-dimensional Friedmann-Lematre-Robertson-Walker (FLRW) model in cylindrical coordinates [9] [10]:

$$
\mathrm{d} s^{2}=\mathcal{W}^{2}\left[\mathrm{e}^{2(\gamma-\psi)}\left(-\mathrm{d} t^{2}+\mathrm{d} r^{2}\right)+\mathrm{e}^{2 \psi} \mathrm{d} z^{2}+r^{2} \mathrm{e}^{-2 \psi} \mathrm{d} \varphi^{2}\right]+\mathrm{d} y^{2},
$$

with $\mathcal{W}$ a warpfactor dependent of $r, t$ and the bulk dimension $y$. The selfgravitating scalar-gauge field, parameterized as

$$
\Phi=\eta X(t, r) \mathrm{e}^{i n \varphi}, \quad A_{\mu}=\frac{1}{\epsilon}[P(t, r)-n] \nabla_{\mu} \varphi,
$$

resides on the brane. $\eta$ is the vacuum expectation value of the scalar field, $n$ the winding number and $\epsilon$ the gauge coupling constant. The winding number (number of jumps in phase of the scalar field when one goes around the flux tube) is related to the quantized flux in the Ginsberg Landau theory of superconductivity (Abrikosov vortices) and the discrete values of the topological charge in the sin-Gordon theory. The exact solution of $\mathcal{W}$ [9] follows from the 5D Einstein equation

$$
{ }^{5} G_{\mu \nu}=-\Lambda_{5}^{5} g_{\mu \nu}+\kappa_{5}^{2} \delta(y)\left(-\Lambda_{4}^{4} g_{\mu \nu}+{ }^{4} T_{\mu \nu}\right),
$$

with $\kappa_{5}=8 \pi^{5} G=8 \pi /{ }^{5} M_{\mathrm{pl}}^{3}, \quad \Lambda_{4}$ the brane tension and $x^{\mu}=\left(t, x^{i}, y\right)$. The ${ }^{5} M_{\mathrm{pl}}$ is the fundamental 5D Planck mass. The scalar-gauge field equations become [4]

$$
D^{\mu} D_{\mu} \Phi=2 \frac{\mathrm{d} V}{\mathrm{~d} \Phi^{*}}, \quad{ }^{4} \nabla^{\mu} F_{v \mu}=\frac{1}{2} i \epsilon\left(\Phi\left(D_{v} \Phi\right)^{*}-\Phi^{*} D_{v} \Phi\right),
$$

with $D_{\mu} \Phi \equiv{ }^{4} \nabla_{\mu} \Phi+i \epsilon A_{\mu} \Phi, \quad{ }^{4} \nabla_{\mu}$ the covariant derivative with respect to ${ }^{4} g_{\mu v}$ and $V$ the potential of the Abelian Higgs model. The star represents the complex conjugated. $F_{\mu \nu}$ is the Maxwell tensor. The modified Einstein equations become [20]

$$
{ }^{4} G_{\mu v}=-\Lambda_{\text {eff }}^{4} g_{\mu v}+\kappa_{4}^{24} T_{\mu v}+\kappa_{5}^{4} \mathcal{S}_{\mu \nu}-\mathcal{E}_{\mu \nu},
$$


with ${ }^{4} G_{\mu v}$ the Einstein tensor calculated on the brane metric ${ }^{4} g_{\mu v}={ }^{5} g_{\mu v}-n_{\mu} n_{v}$ and $n_{\mu}$ the unit vector normal to the brane. We will consider here $\Lambda_{\text {eff }}=0$, so we are dealing with the RS-fine tuning condition [15]. The last two terms on the righthand side of Equation (5) represent the quadratic contribution of the energy-momentum tensor and the electric part of the five dimensional Weyl tensor respectively. All standard model fields are bound to the brane, although gravity may propagate into the extra dimension. Is is obvious, that the cosmic string can build up a huge mass $G \mu \gg 1$ by the warp factor and can induce massive KK-modes felt on the brane. The warp factor causes perturbations to be damped as they move away from the brane, so gravity looks four dimensional, at least perturbatively, to a brane world observer. Brane world models can also explain the acceleration of the universe without the need of a cosmological constant [18]. Disturbances can survive the natural damping by expansion of the universe due to the warp factor. This effect was also found numerically [9].

Here we will consider the modified cosmic string features on the warped spacetime Equation (1) and use the multiple-scale approximation [23] [24] [25] in order to find partial differential equations (PDE's) for the perturbations to second order of the metric and matter fields. This extends our previous research [10].

Let us expand the metric field and the scalar-gauge fields in the multiple-scale scheme

$$
\begin{aligned}
g_{\mu v} & =\bar{g}_{\mu v}(x)+\frac{1}{\omega} h_{\mu v}(x, \xi, \chi, \cdots)+\frac{1}{\omega^{2}} k_{\mu v}(x, \xi, \chi, \cdots)+\cdots, \\
A_{\mu} & =\bar{A}_{\mu}(x)+\frac{1}{\omega} B_{\mu}(x, \xi, \chi, \cdots)+\frac{1}{\omega^{2}} C_{\mu}(x, \xi, \chi, \cdots)+\cdots, \\
\Phi & =\bar{\Phi}(x)+\frac{1}{\omega} \Psi(x, \xi, \chi, \cdots)+\frac{1}{\omega^{2}} \Xi((x, \xi, \chi, \cdots)+\cdots,
\end{aligned}
$$

with $\bar{g}_{\mu v}$ the background metric and $\bar{\Phi}, \bar{A}_{\mu}$ the background scalar and gauge fields. For the scalar field we take different winding numbers, so different magnetic flux quantization for the background field and higher order perturbations. We define $\bar{\Phi}=\eta \bar{X}(t, r) \mathrm{e}^{i n_{1} \varphi}, \Psi=Y(t, r, \xi) \mathrm{e}^{i n_{2} \varphi}$ and

$\Xi=Z(t, r, \xi) \mathrm{e}^{i n_{3} \varphi}$. So we break up the original vortex with winding number $\mathrm{n}$ in our case in three strings with winding numbers $n_{1}, n_{2}$ and $n_{3}$. One can prove [26] that this breakup remains stable if the gauge to scalar mass is $>1$. In our case stability will be guaranteed by inverse powers of the warp factor. Further, we parametrize $B_{\mu}=\left[B_{0}, B_{0}, 0, B, 0\right], C_{\mu}=\left[C_{0}, C_{0}, 0, C, 0\right]$, which will fulfil the highest order perturbation equation of the gauge field as we shall see. Rapid perturbations occur in the direction of the wave vector $l_{\mu} \equiv \frac{\partial \Theta}{\partial x^{\mu}}$. We define

$$
\frac{\mathrm{d} g_{\mu v}}{\mathrm{~d} x^{\sigma}}=g_{\mu v, \sigma}+\omega l_{\sigma} \dot{g}_{\mu v} \quad g_{\mu v, \sigma} \equiv \frac{\partial g_{\mu v}}{\partial x^{\sigma}} \quad \dot{g}_{\mu v} \equiv \frac{\partial g_{\mu v}}{\partial \xi},
$$

Substituting the expansions into the effective Einstein and matter field equations, one obtains a set of PDE's for $\bar{g}_{\mu \nu}, h_{\mu \nu}, k_{\mu v}$ and the scalar gauge fields $\bar{\Phi}, \Psi, \Xi, \bar{A}_{\mu}, B_{\mu}$ and $C_{\mu}$. The perturbations can be $\varphi$-dependent. 
The Einstein equations in subsequent orders of approximation become

$$
\begin{aligned}
& \underline{\omega^{(-1)}}:{ }^{4} G_{\mu \nu}^{(-1)}=-\mathcal{E}_{\mu \nu}^{(-1)}, \\
& \underline{\omega^{(0)}}:{ }^{4} \bar{G}_{\mu \nu}+{ }^{4} G_{\mu \nu}^{(0)}=\kappa_{4}^{2}\left({ }^{4} \bar{T}_{\mu \nu}+{ }^{4} T_{\mu \nu}^{(0)}\right)+\kappa_{5}^{4}\left(\mathcal{S}_{\mu \nu}+\mathcal{S}_{\mu \nu}^{(0)}\right)-\mathcal{E}_{\mu \nu}-\mathcal{E}_{\mu \nu}^{(0)}, \\
& \underline{\omega^{(1)}}:{ }^{4} G_{\mu \nu}^{(1)}=\kappa_{4}^{24} T_{\mu \nu}^{(1)}+\kappa_{5}^{4} \mathcal{S}_{\mu \nu}^{(1)}-\mathcal{E}_{\mu \nu}^{(1)} .
\end{aligned}
$$

We will consider here the equations up to order $\omega^{(1)}$. We used the notation for the several terms in the expansion of a tensor, vector or scalar:

$V_{i}=\omega V^{(-1)}+\bar{V}_{i}+V_{i}^{(0)}+\frac{1}{\omega} V_{i}^{(1)}+\cdots$. The contribution from the bulk space, $\mathcal{E}_{\mu \nu}$, must be calculated with the 5D Riemann tensor. If we consider $l_{\mu} l^{\mu}=0$, i.e., the Eikonal equation (which follows from the $\omega^{(-1)}$ scalar equation), then one obtains from Equation (8) a set of restrictions on $h_{\mu v}$, such as the "gauge condition" $l^{\alpha}\left(\ddot{h}_{\alpha \nu}-\frac{1}{2} \bar{g}_{\alpha \nu} \ddot{h}\right)=0$. Let us consider as a simplified case $l_{\mu}=[1,1,0,0,0]$. Then we let survive $h_{11}, h_{13}, h_{14}, h_{44}$ and $h_{55}$ as independent first order perturbations of the metric.

\section{The Metric Perturbations up to Second Order}

The PDE's for the background fields $\bar{W}_{1}, \bar{\psi}, \bar{\gamma}$ and the first order perturbations $h_{\mu \nu}$ can be derived from Equation (9)and were found in [10]. By integrating Equation (10) with respect to $\xi$, one obtains

$$
{ }^{4} \bar{G}_{\mu \nu}=\kappa_{4}^{24} \bar{T}_{\mu \nu}+\kappa_{5}^{4} \mathcal{S}_{\mu \nu}-\mathcal{E}_{\mu \nu}+\frac{1}{\tau} \int\left(\kappa_{4}^{2} T_{\mu \nu}^{(0)}+\kappa_{5}^{4} \mathcal{S}_{\mu \nu}^{(0)}-{ }^{4} G_{\mu \nu}^{(0)}-\mathcal{E}_{\mu \nu}^{(0)}\right) \mathrm{d} \xi,
$$

with $\tau$ the period of the rapid variation. The last two terms on the right hand side of Equation (11) can be interpreted as "back-reaction" contribution of the KK-modes of the perturbative 5D graviton and can act as a cosmological constant. These equations can be used to eliminate the background fields from the first order PDE's. One then obtains propagation equations for the first order perturbations. For completeness we collected them in appendix A. From these equations one observes that to first order there is an interaction between the high-frequency perturbations from the bulk, the matter fields on the brane and the evolution of $\dot{h}_{i j}$, also found in the numerical solution [9]. The bulk contribution $\dot{h}_{55}$ is amplified by $\bar{W}_{1}^{2}$. It is a reflection of the massive KK modes felt on the brane. The most interesting equation is the differential equation for $\dot{h}_{14}$, i.e., the $(t, \varphi)$ component, Equation (24). It triggers the $\varphi$ dependent disturbances. The $\sin \left[\left(n_{2}-n_{1}\right) \varphi\right]$-term, amplified by warp factor $\bar{W}_{1}$, can have extremal values on $[0, \pi]$, if we choose, for example, $\left(n_{2}-n_{1}\right)=2$. We then have the term $\cos 2 \varphi$, which has two extremal values on $[0, \pi] \bmod \left(\frac{1}{2} \pi\right)$ (also found in [10]).

Let us now investigate the higher order equations in $\omega$, i.e., Equation (10), which will provide us first order equations of $\partial_{t} \dot{k}_{\mu \nu}$ and second order eq- 
uations for $\partial_{t t} h_{\mu v}$. With the help of an algebraic manipulation program, one obtains, for example, the equations for $\partial_{t} \dot{k}_{14}$ and $\partial_{t} \dot{k}_{55}$, were we took for the moment $k_{23}=k_{13}, k_{24}=k_{14}, k_{34}=0$ and $k_{12}=\frac{1}{2}\left(k_{11}+k_{22}\right)$. See Appendix B. We observe in Equation (33) again a $\cos \left[\left(n_{2}-n_{1}\right) \varphi\right]$-term amplified by the warp factor. In the equation Equation (34) for $\partial_{t} \dot{k}_{14}$, there appears besides the term $\sin \left[\left(n_{2}-n_{1}\right) \varphi\right]$, also a $\sin \left[\left(n_{3}-n_{1}\right) \varphi\right]$-term in connection with the second order perturbation $\dot{Z}$, amplified by the warp factor. In the equation for the first order counterpart equation, i.e., Equation (24), there is only the $\sin \left[\left(n_{2}-n_{1}\right) \varphi\right]$-term. So if we take in Equation (34) for $\left(n_{3}-n_{1}\right)=4$ (and $\left.\left(n_{2}-n_{1}\right)=2\right)$, then the maxima in $\varphi$ of these two terms belonging to the perturbations of first and second order respectively, are out-of-phase. In the next section this will also become clear by considering the energy-current components of the energy momentum tensor. From Equation (33) and Equation (26) we can obtain a second order PDE for $h_{55}$ if we impose constraint conditions on $\dot{k}_{55}$ or integrate Equation (33) with respect to $\xi$. This can also be done for the other components. So we can construct with the multiple-scale method uniformly valid wavelike approximations to solutions of perturbation problems without without "resonance" interactions between the consecutive orders of perturbation theory. This result is related to the Cauchy problem. In any field theory where there is a gauge freedom (as, for example, in GRT and Maxwell theory), one has to specify gauge conditions in order to determine the dynamical evolution for some initial set of Cauchy data. In Maxwell theory one usually choose the Lorentz gauge. In GRT one has constraint equations because the system is over determined. These constraints are usually PDE's of first order. In our approximation scheme we have Equation (8) which leads to conditions on $\dot{h}_{\mu v}$. The first order equations Equations (23)-(27) can also be considered as constraint equations for the second order wave equations for $h_{\mu v}$, as example, in the case of $h_{55}$. So we can construct a dynamical evolution of the system of equations which fulfil the Cauchy data.

We shall see in the next sections how this result can be applied in context with the recently observed alignment of the polarization vectors of quasars over large distances and the discreteness in the azimuthal dependency of the polarization axes.

\section{The Matter Field Equations and the Energy-Momentum Tensor Expansion}

From Equation (4) we obtain, after substituting the expansions of Equation (6), from the $\omega^{(-1)}$ equations the conditions $l_{\mu} l^{\mu} \ddot{\Psi}=0$ and $l^{\mu} \ddot{B}_{\mu}=0$. So we will parameterize $B_{\mu}=\left[B_{0}, B_{0}, 0, B, 0\right]$ and $I^{\mu} I_{\mu}=0$, otherwise $\Psi$ must be zero. From the first order equation of the scalar field we obtain

$$
\bar{D}^{\alpha} \bar{D}_{\alpha} \bar{\Phi}-\frac{1}{2} \beta \bar{\Phi}\left(\bar{\Phi} \bar{\Phi}^{*}-\eta^{2}\right)=\frac{1}{\tau} \int\left(h^{\mu \nu} l_{\mu} l_{\nu} \ddot{\Psi}+\bar{g}^{\mu \nu} \Gamma_{\mu \nu}^{\alpha(0)} l_{\alpha} \dot{\Psi}\right) \mathrm{d} \xi,
$$

where we have integrated the equation with respect to $\xi$. On the right hand 
side we see again the high-frequency contribution to the field equation. In our case, this back reaction term turns out to be zero. So the first order equation is just the unperturbed equation for $\bar{X}$. See Equation (28) of Appendix A. This also holds for the equation for $\bar{A}_{\mu}$. See Equation (29) of appendix A. If we substitute back the integrated equations into the original equations, we then obtain the first order perturbations (for $l^{\alpha} C_{\alpha}=0 ; \Psi=Y(t, r, \xi) \mathrm{e}^{i n_{2} \varphi}$ ). See Equation (30)-Equation (32) of Appendix A. Further, we used $l^{\alpha} \bar{A}_{\alpha}=0$, otherwise the real and imaginary parts of $\dot{\Psi}$ interact as the propagation progresses. Again, there appears a $\varphi$-dependent term in the propagation equation for $B_{0}$, amplified by $\bar{W}_{1}$. This deviation from axially symmetry was also found by [23]. More insight in this $\varphi$-dependency can be obtained by studying the second-order matter field equations. One obtains for $\dot{Z}$ and $\dot{C}$ again first order differential equations. See Appendix B. We used the fact that the complex conjugate of the full complex second order scalar equation also must be satisfied. The appearance of the terms $\cos \left[\left(n_{3}-n_{2}\right) \varphi\right], \cos \left[\left(n_{3}-n_{1}\right) \varphi\right]$ and $\cos \left[\left(n_{3}+n_{2}-2 n_{1}\right) \varphi\right]$ will contribute to the next order modes of maxima in $\varphi$ dependent disturbances.

We can obtain, as in the case of the second order metric components, again second order PDE's for $\partial_{t t} B$ and $\partial_{t t} Y$ by suitable constraints on $C$ and $Z$ or integration with respect to $\xi$. After some rearrangement of Equation (35) for example, we get the wave equation for $Y$ (after suitable constraints on $\dot{Z}$ ).

$$
\begin{aligned}
\partial_{t t} Y= & \partial_{r r} Y+\frac{\partial_{r} Y}{r}-\frac{\mathrm{e}^{2 \bar{\gamma}}}{r^{2}} Y\left(n_{2}-n_{1}+\bar{P}\right)^{2}-\beta \bar{W}_{1}^{2} \mathrm{e}^{2 \bar{\gamma}-2 \bar{\psi}} Y \bar{X}^{2} \\
& +2 \frac{\partial_{r} Y \partial_{r} \bar{W}_{1}-\partial_{t} Y \partial_{t} \bar{W}_{1}}{\bar{W}_{1}}+2 \mathrm{e}^{2 \bar{\psi}} \frac{\dot{Y}}{\bar{W}_{1}^{2} r^{2}}\left(\partial_{r} \bar{\psi}-\partial_{t} \bar{\psi}-\frac{1}{2 r}\right) h_{44} \\
& +2 \mathrm{e}^{2 \bar{\psi}-2 \bar{\gamma}} \frac{\dot{Y}}{\bar{W}_{1}^{2}}\left(\partial_{t} \bar{\psi}-\partial_{r} \bar{\psi}+\partial_{r} \bar{\gamma}-\partial_{t} \bar{\gamma}+\frac{\partial_{r} \bar{W}_{1}-\partial_{t} \bar{W}_{1}}{\bar{W}_{1}}\right) h_{11} \\
& +2 \mathrm{e}^{2 \bar{\psi}-2 \bar{\gamma}} \frac{\partial_{r} \dot{Y}-\partial_{r} \dot{Y}}{\bar{W}_{1}^{2}} h_{11}+\frac{\mathrm{e}^{2 \bar{\psi}-2 \bar{\gamma}}}{\bar{W}_{1}^{2}}\left(\partial_{t} Y-\partial_{t} Y\right) \dot{h}_{11} \\
& +\frac{\dot{Y}^{2 \bar{\psi}-2 \bar{\gamma}}}{\bar{W}_{1}^{2}}\left(\partial_{t} h_{11}-\partial_{r} h_{11}\right)+\mathcal{A} \cos \left[\left(n_{2}-n_{1}\right) \varphi\right] \\
& -\frac{1}{2} \beta Y \bar{W}_{1}^{2} \bar{X}^{2} \mathrm{e}^{2 \bar{\gamma}-2 \bar{\psi}} \cos \left[2\left(n_{2}-n_{1}\right) \varphi\right] \\
& +2\left[\partial_{r} \dot{Z}-\partial_{t} \dot{Z}+\left(\frac{\partial_{r} \bar{W}_{1}-\partial_{t} \bar{W}_{1}}{\bar{W}_{1}}+\frac{1}{2 r}\right) \dot{Z}\right] \cos \left[\left(n_{3}-n_{2}\right) \varphi\right],
\end{aligned}
$$

where $\mathcal{A}$ is an expression in backgrounds fields, $h_{11}$ and $h_{44}$. We have again two periodic functions $\cos \left[2\left(n_{2}-n_{1}\right) \varphi\right]$ and $\cos \left[\left(n_{2}-n_{1}\right) \varphi\right]$ with frequency difference of a factor two and where one of the functions is amplified by $\bar{W}_{1}^{2}$. It will be necessary to study these equations numerically in order to compare the amplitudes of these two periodic functions with those of the azimuthal-angle dependent maxima of the quasar polarization alignment.

The second order equation for $B_{0}$ can be obtained from the sum of the $t$ and $r$-components of the second order gauge field equations. 
We can calculate the three first terms of the energy momentum tensor ${ }^{4} T_{\mu v}$. See Appendix C. In ${ }^{4} T_{t t}^{(1)}$ there appears, for $\left(n_{2}-n_{1}\right)=2$ and $\left(n_{3}-n_{1}\right)=4$, the terms $\cos (2 \varphi), \sin (2 \varphi)$ and $\cos (4 \varphi)$, while in the first order term, Equation (41), there is only the $\cos (2 \varphi)$. This is also true for the energycurrent components ${ }^{4} T_{t \varphi}^{(0)}$ and ${ }^{4} T_{t \varphi}^{(1)}$. In the energy momentum tensor component ${ }^{4} T_{t t}^{(1)}$ there is also a term proportional $\left(n_{2}-n_{1}+\bar{P}\right)$. In the next order ${ }^{4} T_{t t}^{(2)}$ there will be terms proportional with higher orders of $\left(n_{2}-n_{1}+\bar{P}\right)$. These higher order terms will be suppressed by the warp factor, so the vortex will not become unstable as is the case when one breakup the vortex string in multiple flux [27].

The most interesting behavior arises in the angular component ${ }^{4} T_{\varphi \varphi}$, i.e., Equation (43)-Equation (45). As already noticed by Laguna-Castillo and Matzner [5], ${ }^{4} \bar{T}_{\varphi \varphi}$ can alternate in sign dependent of the gauge to scalar mass. This can also happen dynamically [9]. In the next order ${ }^{4} T_{\varphi \varphi}^{(0)}$ we have the $\dot{Y}$ contribution in front of $\cos [2 \varphi]$ and in the next order ${ }^{4} T_{\varphi \varphi}^{(1)}$ the $\dot{Z}$ contribution in front of $\cos [4 \varphi]$ (for the chosen values of $n_{i}$ as above). So the doubling of the frequency in obvious. From the expression for ${ }^{4} T_{z z}^{(0)}$, Equation (46), we see that the pressure in the z-direction is again dominated by the $\cos \left[\left(n_{2}-n_{1}\right) \varphi\right]$, because the second term is suppressed by $\bar{W}_{1}$. There is, however, a peculiar side effect: the term $\left(\partial_{t} \bar{X}-\partial_{r} \bar{X}\right)$ can change sign dynamically. A numerical solution can give a decisive answer.

There is a relation between the phase freedom $\mathrm{e}^{\text {in } \varphi}$ of our scalar field and the secular instability of an initially quasi-stationary axially symmetric configuration caused by radiative reaction. The small non-axially symmetric deformations turn out to be of the form $\mathrm{e}^{\mathrm{im} \varphi}$ with $\mathrm{m}$ an integer [28]. This broken symmetry, described by the inverse of the angular momentum $\mathrm{J}$, is comparable with the symmetry breaking of the Higgs field considered in our model. An axially symmetric systems is invariant under rotations in two dimensions, the $\mathrm{SO}(2)$ group. The breaking of this symmetry can be expressed in the equatorial eccentricity. The particular orientation of the ellipsoid in the $(x-y)$ frame can be expressed through the azimuthal angle $\varphi$. This discrete change into non-axially symmetry must be cancelled by emission of gravitational energy (and is amplified in our model by the 5D contribution), otherwise we are saddled with a helical time coordinate, $t \rightarrow t+J \varphi$ and must give up Lorentz invariance. This is clear from the fact that our metric will then possess a $g_{t \varphi}$ term. The angular momentum in $(x-y)$ plane is determined by the currents of the momentum density, $\sim x^{\rho} T^{v \mu}-x^{\nu} T^{\rho \mu}$ and can be calculated in our case with the off-diagonal components of $T_{\mu \nu}$ of Appendix C. For example $J \sim \epsilon_{i j} \int \mathrm{d}^{2} x\left(x^{i} T^{0 j}-x^{j} T^{0 i}\right)$.

\section{Quasar Polarization Alignment and Scale-Invariance}

In order to explain the recently found large-scale alignments of the polarization vectors of quasars in LQGs at cosmological redshifts $z \sim 1.5$ by cosmic strings, it would be desirable to find a kind of scale (conformal)-invariance, because it is conjectured that cosmic string networks evolve scale-invariant just after the 
radiation dominated era of our universe. The intricate features of the polarization axes alignments of the quasars in LQGs show at least a kind of coevolution at very large scales [22], so a study of conformal symmetry of our model could be of interest.

Gravity theory invariant under $g_{\mu v}(x) \rightarrow \Omega^{2}(x) g_{\mu v}(x)$ is local conformal invariant and must be spontaneously broken because our world appears not to be scale invariant [29] [30]. Let us rewrite our spacetime of Equation (1) with $\psi=\gamma=0$

$$
\mathrm{ds} s^{2}=\Omega^{2}\left[-\mathrm{d} t^{2}+\mathrm{d} r^{2}+\mathrm{d} z^{2}+r^{2} \mathrm{~d} \varphi^{2}+\frac{1}{\Omega^{2}} \mathrm{~d} y^{2}\right] .
$$

where we renamed the warp factor as $\Omega$. If we consider the $(r, t)$-dependent part of $\Omega$ and consider the flat (brane) case of the metric Equation (1),

$$
\hat{\mathrm{d}} s^{2}=-\mathrm{d} t^{2}+\mathrm{d} r^{2}+\mathrm{d} z^{2}+r^{2} \mathrm{~d} \varphi^{2}+\frac{1}{\Omega^{2}} \mathrm{~d} y^{2} .
$$

we then obtain for the Ricci scalar

$$
{ }^{5} \hat{R}=\frac{2}{\Omega}\left(\partial_{r r} \Omega-\partial_{t t} \Omega+\frac{\partial_{r} \Omega}{r}\right)+\frac{4}{\Omega^{2}}\left(\partial_{t} \Omega^{2}-\partial_{r} \Omega^{2}\right) .
$$

The Ricci scalar transforms under $g_{\mu \nu}(x) \rightarrow \Omega^{2}(x) g_{\mu \nu}(x)$ as [31]

$$
\begin{aligned}
& { }^{5} \hat{R} \rightarrow \frac{1}{\Omega^{2}}\left[{ }^{5} \hat{R}-\frac{4 \hat{g}^{\mu \nu} \hat{\nabla}_{\mu} \Omega \hat{\nabla}_{\nu} \Omega}{\Omega^{2}}-\frac{8 \hat{g}^{\mu \nu} \hat{\nabla}_{\mu} \hat{\nabla}_{v} \Omega}{\Omega}\right] \\
& =\frac{1}{\Omega^{2}}\left[{ }^{5} \hat{R}+\frac{4\left(\partial_{r} \Omega^{2}-\partial_{t} \Omega^{2}\right)}{\Omega^{2}}+\frac{8\left(\partial_{t t} \Omega-\partial_{r r} \Omega-\frac{1}{r} \partial_{r} \Omega\right)}{\Omega}\right] .
\end{aligned}
$$

So for conformal invariancy of ${ }^{5} \hat{R}$, the second term on the right hand side of Equation (17) must vanish. For ${ }^{5} \hat{R}=0$ we then find

$$
\partial_{t t} \Omega-\partial_{r r} \Omega-\frac{1}{r} \partial_{r} \Omega=0
$$

with constraint equations $\partial_{t} \Omega^{2}-\partial_{r} \Omega^{2}=0$. Equation (18) is just the equation of a vibrating circular drum. The general solution for the boundary conditions $\Omega(r, 0)=f(r), \partial_{t} \Omega(r, 0)=0$ is

$$
\Omega_{n}(r, t)=\left(A \cos \left(c_{n} t\right)+B \sin \left(c_{n} t\right)\right)\left(J_{0}\left(c_{n} r\right)+Y_{0}\left(c_{n} r\right)\right),
$$

with $J_{0}, Y_{0}$ Bessel functions and $C_{n}$ coefficients dependent of $f(r)$. These solutions represent for suitable boundary conditions, the standing normal modes of the brane in the vacuum case. In general, $\Omega$ can also depend on the azimuthal angle. This dependency is found, in our non-vacuum situation, in the preceding sections in the multiple-scale approximation. So one could conclude that the warp factor in the vacuum case fulfils a scalar wave equations representing fluctuations of the brane in the ground state. It represents the amount of local "stretching" of the $4 \mathrm{D}$ geometry. In the non-vacuum case, with the $U(1)$ 
scalar gauge field in the brane, one can try to formulate again the conformal invariance. This is a peculiar issue in theoretical physics till now. Einstein equations and the scalar equation (Klein-Gordon equation)) are not conformally invariant. One has to modify Einstein's equations to make it conformally invariant and make the energy momentum tensor traceless [31] [32]. Our $\Omega$ field can play a crucial role in this context if one introduces an unavoidable dilaton field.

\section{Conclusions}

It is found on a five dimensional warped brane world spacetime, using a multiple-scale approximation scheme, that to second order the metric and scalar gauge field show a spectrum of azimuthal-angle dependent wavelike modes with extremal values dependent of the winding numbers of the background, first and second order perturbations of the scalar field.

In four dimensional models, this local field theory admits vortex-like behavior and is a generalization of the Ginzburg-Landau theory of superconductivity. A lattice of Abrikosov vortices can be formed, carrying a quantized flux dependent of the winding number or "vortex charge" $n$. Vortices with $n>1$ are unlikely, since the energy is reduced if they split up into single vortices. However, in the general relativistic case, gravity comes into play and the energy of the configuration of the vortices must be calculated covariantly by means of the energy momentum tensor. This general relativistic vortex solution (cosmic string) can build up a huge mass per unit length in the bulk and can induce massive Kaluza-Klein modes felt on the brane, where the standard model fields reside. Disturbances don't fade away during the expansion of the universe due to the warp factor.

The jump in the phase of the scalar field is related to the secular instability of the initially stationary axially symmetric configuration caused by the radiation reaction. The breaking of the axially symmetry, described by the inverse of the angular momentum, is $\sim \mathrm{e}^{\mathrm{i} m \varphi}$ ( $m$ an integer and $\varphi$ the azimuthal angle), comparable with the symmetry breaking of the scalar field. The recovery of the $\mathrm{SO}(2)$ symmetry from the equatorial eccentricity is triggered by the emission of gravitational waves. Our model can be used to explain the mysterious alignment of quasar axes with the large-scale structure of our universe and can serve as evidence for the existence of cosmic strings. The found fractional azimuthal structure is comparable with the angle-preferences of the polarization axes of quasars.

There is a strong evidence of scaling (conformal) behaviour of long nonintercommuting cosmic strings networks during the radiation-dominated era. High or low initial string densities tend toward a fixed scaling value. However, standard cosmology, constraints such strings to be very light and will fade away (or disappear by the forming of closed loops). In our model they can survive by the warp factor. This fact makes the comparison with the alignment of quasars possible. So it would be desirable to have also a scale-invariant alignment 
structure. This is currently under study.

\section{References}

[1] Vilenkin, A. and Shellard, E.P.S. (1994) Cosmic Strings and Other Topological Defects. Cambridge University press, Cambridge, UK.

[2] Anderson, M.R. (2003) The Mathematical Theory of Cosmic Strings. IoP Publishing, Bistol. https://doi.org/10.1887/0750301600

[3] Nielsen, H.B. and Olesen, P. (1973) Nuclear Physics B, 61, 45.

[4] Garfinkle, D. (1985) Physical Review D, 32, 1323. https://doi.org/10.1103/PhysRevD.32.1323

[5] Laguna-Castilo, P. and Matzner, R.A. (1987) Physical Review D, 36, 3663. https://doi.org/10.1103/PhysRevD.36.3663

[6] Gregory, R. (1989) Physical Review D, 39, 2108. https://doi.org/10.1103/PhysRevD.39.2108

[7] Hutsemekers, D., Braibant, L., Pelgrims, V. and Sluse, D. (2014) Astronomy \& Astrophysics, 572, A18. https://doi.org/10.1051/0004-6361/201424631

[8] Taylor, A.R. and Jagannathan, P. (2016) Monthly Notices of the Royal Astronomical Society, 459, L36.

[9] Slagter, R.J. and Pan, S. (2016) Foundations of Physics, 46, 1075. https://doi.org/10.1007/s10701-016-0002-2

[10] Slagter, R.J. (2016) Journal of Modern Physics, 7, 501. https://doi.org/10.4236/jmp.2016.76052

[11] Laguna-Castillo, P. and Garfinkle, D. (1989) Physical Review D, 40, 1011-1016. https://doi.org/10.1103/PhysRevD.40.1011

[12] Arkani-Hamed, N., Dimopoulos, S. and Dvali, G. (1992) Physics Letters B, 429, 263-272. https://doi.org/10.1016/S0370-2693(98)00466-3

[13] Arkani-Hamed, N., Dimopoulos, S. and Dvali, G. (1999) Physical Review D, 59, Article ID: 086004.

[14] Antoniadis, I., Arkani-Hamed, N., Dimopoulos, S. and Dvali, G. (1998) Physics Letters B, 436, 257-263. https://doi.org/10.1016/S0370-2693(98)00860-0

[15] Randall, L. and Sundrum, R. (1999) Physical Review Letters, 83, 3370-3373. https://doi.org/10.1103/PhysRevLett.83.3370

[16] Randall, L. and Sundrum, R. (1999) Physical Review Letters, 83, 4690-4693. https://doi.org/10.1103/PhysRevLett.83.4690

[17] Maartens, R. (2007) Journal of Physics: Conference Series, 68, Article ID: 012046. https://doi.org/10.1088/1742-6596/68/1/012046

[18] Maartens, R. (2007) Lecture Notes in Physics, 720, 323-332.

[19] Maartens, R. and Koyama, K. (2010) Living Reviews in Relativity, 13, 5. https://doi.org/10.12942/lrr-2010-5

[20] Shiromizu, T., Maeda, K. and Sasaki, M. (2000) Physical Review D, 62, Article ID: 024012.

[21] Slagter, R.J. (2014) International Journal of Modern Physics D, 10, 1237.

[22] Pelgrims, V. (2016) ArXiv:astro-ph/160405141v1.

[23] Choquet-Bruhat, Y. (1969) Communications in Mathematical Physics, 12, 16-35. https://doi.org/10.1007/BF01646432

[24] Choquet-Bruhat, Y. (1977) General Relativity and Gravitation, 8, 561-571. 
https://doi.org/10.1007/BF00756307

[25] Slagter, R.J. (1986) Astrophysical Journal, 307, 20-29.

https://doi.org/10.1086/164388

[26] Bogomol'nyi, E. (1976) Soviet Journal of Nuclear Physics, 24, 449-454.

[27] Felsager, B. (1987) Geometry, Particles and Fields. Odense University Press, Odense.

[28] Chandrasekhar, S. and Lebovitz, N.R. (1973) Astrophysical Journal, 185, 19-30. https://doi.org/10.1086/152393

[29] 'T Hooft, G. (2014) ArXiv: gr-qc/14106675v3.

[30] 'T Hooft, G. (2015) ArXiv: gr-qc/151104427v1.

[31] Wald, R.W. (1984) General Relativity. The University of Chicago Press, Chicago. https://doi.org/10.7208/chicago/9780226870373.001.0001

[32] Maldacena, J. (2011) ArXiv: gr-qc/11055632v2. 


\section{Appendix}

\section{A. The Background and First Order Perturbation Equations}

In an earlier work [10] we obtained the equations for the background metric components $\left(\bar{W}_{1}, \bar{\psi}, \bar{\gamma}\right)$, background matter fields $\bar{X}, \bar{Y}$ and first order approximation equations of $\dot{h}_{13}, \dot{h}_{14}, \dot{h}_{11}, \dot{h}_{44}, \dot{Y}, \dot{B}$ and $\dot{B}_{0}$. They are

$$
\begin{aligned}
& \partial_{t t}^{2} \bar{W}_{1}=-\partial_{r r}^{2} \bar{W}_{1}+\frac{2}{\bar{W}_{1}}\left(\partial_{t} \bar{W}_{1}^{2}+\partial_{r} \bar{W}_{1}^{2}\right)-\bar{W}_{1}\left(\partial_{t} \bar{\psi}^{2}+\partial_{r} \bar{\psi}^{2}\right)+\frac{\bar{W}_{1}}{r}\left(\partial_{r} \bar{\gamma}-\partial_{t} \bar{\gamma}\right) \\
& +2\left(\partial_{r} \bar{W}_{1}-\partial_{t} \bar{W}_{1}\right)\left(\partial_{t} \bar{\psi}-\partial_{r} \bar{\psi}+\partial_{r} \bar{\gamma}-\partial_{t} \bar{\gamma}\right)+2 \bar{W}_{1} \partial_{r} \bar{\psi} \partial_{t} \bar{\psi} \\
& -4 \frac{\partial_{r} \bar{W}_{1} \partial_{t} \bar{W}_{1}}{\bar{W}_{1}}+2 \partial_{r t} \bar{W}_{1}-\frac{3}{4} \kappa_{4}^{2}\left(\mathrm{e}^{2 \bar{\psi}} \frac{\left(\partial_{t} \bar{P}-\partial_{r} \bar{P}\right)^{2}}{\bar{W}_{1} r^{2} \epsilon^{2}}+\bar{W}_{1}\left(\partial_{t} \bar{X}-\partial_{r} \bar{X}\right)^{2}\right), \\
& \partial_{t t} \bar{\psi}=\partial_{r r} \bar{\psi}+\frac{\partial_{r} \bar{\psi}}{r}+\frac{2}{\bar{W}_{1}}\left(\partial_{r} \bar{W}_{1} \partial_{r} \bar{\psi}-\partial_{t} \bar{W}_{1} \partial_{t} \bar{\psi}\right)-\frac{\partial_{r} \bar{W}_{1}}{r \bar{W}_{1}} \\
& +\frac{3 \mathrm{e}^{2 \bar{\psi}}}{4 \bar{W}_{1}^{2} r^{2} \epsilon^{2}} \kappa_{4}^{2}\left(\partial_{t} \bar{P}^{2}-\partial_{r} \bar{P}^{2}-\bar{W}_{1}^{2} \epsilon^{2} \bar{X}^{2} \bar{P}^{2} \mathrm{e}^{2 \bar{\gamma}-2 \bar{\psi}}\right), \\
& \partial_{t} \bar{\gamma}=\partial_{r} \bar{\gamma}+\frac{1}{\partial_{t} \bar{W}_{1}-\partial_{r} \bar{W}_{1}-\frac{\bar{W}_{1}}{2 r}}\left[\frac{1}{2} \bar{W}_{1}\left(\partial_{t} \bar{\psi}-\partial_{r} \bar{\psi}\right)^{2}+\frac{\partial_{r} \bar{W}_{1}}{r}-\partial_{t r} \bar{W}_{1}\right. \\
& -\frac{\partial_{r} \bar{W}_{1}^{2}+3 \partial_{t} \bar{W}_{1}^{2}}{2 \bar{W}_{1}}+\partial_{r r} \bar{W}_{1}+\frac{2 \partial_{r} \bar{W}_{1} \partial_{t} \bar{W}_{1}}{\bar{W}_{1}}+\left(\partial_{r} \bar{W}_{1}-\partial_{t} \bar{W}_{1}\right)\left(\partial_{r} \bar{\psi}-\partial_{t} \bar{\psi}\right) \\
& +\kappa_{4}^{2} \frac{\bar{W}_{1}}{16}\left(7 \partial_{t} \bar{X}^{2}+5 \partial_{r} \bar{X}^{2}-12 \partial_{r} \bar{X} \partial_{t} \bar{X}+5 \mathrm{e}^{2 \bar{\gamma}} \frac{\bar{X}^{2} \bar{P}^{2}}{r^{2}}\right. \\
& \left.\left.+6 \mathrm{e}^{2 \bar{\psi}} \frac{\left(\partial_{r} \bar{P}-\partial_{t} \bar{P}\right)^{2}}{\bar{W}_{1}^{2} r^{2} \epsilon^{2}}+\bar{W}_{1}^{2} \beta \mathrm{e}^{2 \bar{\gamma}-2 \bar{\psi}}\left(\bar{X}^{2}-\eta^{2}\right)^{2}\right)\right] \text {. } \\
& \partial_{t} \dot{h}_{13}=\partial_{r} \dot{h}_{13}+\ddot{k}_{13}-\ddot{k}_{23}+2\left(\frac{\partial_{t} \bar{W}_{1}-\partial_{r} \bar{W}_{1}}{\bar{W}_{1}}+\partial_{t} \bar{\psi}-\partial_{r} \bar{\psi}\right) \dot{h}_{13}, \\
& \partial_{t} \dot{h}_{14}=\partial_{r} \dot{h}_{14}+\ddot{k}_{14}-\ddot{k}_{24}+2\left(\partial_{r} \bar{\psi}-\partial_{t} \bar{\psi}+\frac{\partial_{t} \bar{W}_{1}-\partial_{r} \bar{W}_{1}}{\bar{W}_{1}}-\frac{1}{r}\right) \dot{h}_{14} \\
& +2 \kappa_{4}^{2} \mathrm{e}^{2 \bar{\gamma}-2 \bar{\psi}} \bar{W}_{1}^{2} \overline{X P} \dot{Y} \sin \left[\left(n_{2}-n_{1}\right) \varphi\right]+\partial_{\varphi}\left[\bar{W}_{1}^{2} \mathrm{e}^{2 \bar{\gamma}-2 \bar{\psi}} \dot{h}_{55}-\dot{h}_{11}-\frac{\mathrm{e}^{2 \bar{\gamma}}}{r^{2}} \dot{h}_{44}\right], \\
& \partial_{t} \dot{h}_{11}=\partial_{r} \dot{h}_{11}+\frac{\mathrm{e}^{2 \bar{\gamma}}}{r^{2}}\left(\partial_{r} \bar{\psi}-\partial_{t} \bar{\psi}-\frac{1}{2 r}\right) \dot{h}_{44}+\frac{1}{2}\left(\ddot{k}_{22}+\ddot{k}_{11}\right)-\ddot{k}_{12} \\
& +\frac{2}{\bar{W}_{1}}\left(\partial_{t} \bar{W}_{1}-\partial_{r} \bar{W}_{1}+\bar{W}_{1}\left(\partial_{r} \bar{\psi}-\partial_{t} \bar{\psi}+\partial_{t} \bar{\gamma}-\partial_{r} \bar{\gamma}\right)\right) \dot{h}_{11} \\
& +\mathrm{e}^{2 \bar{\gamma}-2 \bar{\psi}} \bar{W}_{1}^{2}\left[\left(\frac{1}{4 r}+\frac{\partial_{r} \bar{W}_{1}-\partial_{t} \bar{W}_{1}}{2 \bar{W}_{1}}\right) \dot{h}_{55}+\kappa_{4}^{2}\left(\partial_{t} \bar{X}-\partial_{r} \bar{X}\right) \dot{Y}\right] \cos \left[\left(n_{2}-n_{1}\right) \varphi\right], \\
& \partial_{t} \dot{h}_{55}-\partial_{r} \dot{h}_{55}=0 \text {, } \\
& \partial_{t} \dot{h}_{44}=\partial_{r} \dot{h}_{44}+\left(2 \partial_{r} \bar{\psi}-2 \partial_{t} \bar{\psi}-\frac{3}{2 r}+\frac{\partial_{t} \bar{W}_{1}-\partial_{r} \bar{W}_{1}}{\bar{W}_{1}}\right) \dot{h}_{44} \\
& +\frac{\kappa_{4}^{2}}{\epsilon}\left(\partial_{r} \bar{P}-\partial_{t} \bar{P}\right) \dot{B}+\frac{1}{2} \bar{W}_{1}^{2} r^{2} \mathrm{e}^{-2 \bar{\psi}}\left(\partial_{t} \bar{\psi}-\partial_{r} \bar{\psi}+\frac{1}{2 r}\right) \dot{h}_{55} .
\end{aligned}
$$


The background matter fields $(\bar{X}, \bar{P})$ become $[9]$

$$
\begin{gathered}
\partial_{t t} \bar{X}=\partial_{r r} \bar{X}+\frac{\partial_{r} \bar{X}}{r}+2\left(\frac{\partial_{r} \bar{W}_{1} \partial_{r} \bar{X}}{\bar{W}_{1}}-\frac{\partial_{t} \bar{W}_{1} \partial_{t} \bar{X}}{\bar{W}_{1}}\right) \\
-\frac{\mathrm{e}^{2 \bar{\gamma}} \overline{X P}^{2}}{r^{2}}-\frac{1}{2} \bar{W}_{1}^{2} \mathrm{e}^{2 \bar{\gamma}-2 \bar{\psi}} \beta \bar{X}\left(\bar{X}^{2}-\eta^{2}\right), \\
\partial_{t t} \bar{P}=\partial_{r r} \bar{P}-\frac{\partial_{r} \bar{P}}{r}+2\left(\partial_{r} \bar{P} \partial_{r} \bar{\psi}-\partial_{t} \bar{P} \partial_{t} \bar{\psi}\right)-\epsilon^{2} \bar{W}_{1}^{2} \mathrm{e}^{2 \bar{\gamma}-2 \bar{\psi}} \bar{P} \bar{X}^{2},
\end{gathered}
$$

and perturbation equations

$$
\begin{gathered}
\partial_{t} \dot{Y}=\partial_{r} \dot{Y}+\left(\frac{\partial_{r} \bar{W}_{1}-\partial_{t} \bar{W}_{1}}{\bar{W}_{1}}+\frac{1}{2 r}\right) \dot{Y} \\
\partial_{t} \dot{B}=\partial_{r} \dot{B}+\left(\partial_{r} \bar{\psi}-\partial_{t} \bar{\psi}-\frac{1}{2 r}\right) \dot{B}+\frac{\mathrm{e}^{2 \bar{\psi}}\left(\partial_{t} \bar{P}-\partial_{r} \bar{P}\right)}{2 r^{2} \bar{W}_{1}^{2} \epsilon} \dot{h}_{44}, \\
\partial_{t} \dot{B}_{0}=\partial_{r} \dot{B}_{0}-\frac{\mathrm{e}^{2 \bar{\gamma}}}{r^{2}} \partial_{\varphi} \dot{B}+\frac{\mathrm{e}^{2 \bar{\psi}}\left(\partial_{t} \bar{P}-\partial_{r} \bar{P}\right)}{\bar{W}_{1}^{2} r^{2} \epsilon} \dot{h}_{14} \\
+\epsilon \mathrm{e}^{2 \bar{\gamma}-2 \bar{\psi}} \dot{Y} \bar{X} \bar{W}_{1}^{2} \sin \left[\varphi\left(n_{2}-n_{1}\right)\right] .
\end{gathered}
$$

These background equations don't contain perturbations terms, due to our (simplified) gauge conditions.

\section{B. The Second Order Perturbation Equations}

With the help of a algebraic manipulation program, we obtain from Equation (10), for example, the most interesting:

$$
\begin{aligned}
\partial_{t} \dot{k}_{55}= & \partial_{r} \dot{k}_{55}+\frac{1}{2}\left(\partial_{r r} h_{55}-\partial_{t t} h_{55}\right)+\frac{1}{2}\left(\partial_{t} h_{55}-\partial_{r} h_{55}\right) \dot{h}_{55} \\
& +\frac{\mathrm{e}^{2 \bar{\psi}-2 \bar{\gamma}}}{\bar{W}_{1}^{2}}\left(\partial_{t} \dot{h}_{55}-\partial_{r} \dot{h}_{55}\right) h_{11}+\kappa_{4}^{2} \frac{\mathrm{e}^{2 \bar{\gamma}}}{r^{2}} \bar{X} Y\left(2 \bar{P}\left(n_{1}-n_{2}-\bar{P}\right)\right. \\
& \left.+\mathrm{e}^{-2 \bar{\psi}} r^{2} \bar{W}_{1}^{2} \beta\left(\eta^{2}-\bar{X}^{2}\right)\right) \cos \left[\left(n_{2}-n_{1}\right) \varphi\right]+2 \frac{\mathrm{e}^{2 \bar{\psi}}}{\bar{W}_{1}^{2} r^{2}}\left[\kappa_{4}^{2} \frac{\mathrm{e}^{2 \bar{\gamma}}}{r^{2}} \bar{X}^{2} \bar{P}^{2}\right. \\
& +\partial_{r r} \bar{\psi}-\partial_{t t} \bar{\psi}+\frac{\mathrm{e}^{2 \bar{\psi}}}{\bar{W}_{1}^{2} r^{2}}\left(2 \partial_{r} \bar{\psi}-2 \partial_{t} \bar{\psi}+\frac{\partial_{t} \bar{W}_{1}-\partial_{r} \bar{W}_{1}}{\bar{W}_{1}}-\frac{3}{2 r}\right) \dot{h}_{44} \\
& \left.+\frac{2\left(\partial_{t} \bar{W}_{1} \partial_{t} \bar{\psi}-\partial_{r} \bar{W}_{1} \partial_{r} \bar{\psi}\right)}{\bar{W}_{1}}+\frac{\partial_{r} \bar{\psi}}{r}-\frac{\partial_{r} \bar{W}_{1}}{\bar{W}_{1} r}\right] h_{44} \\
& +2 \frac{\mathrm{e}^{2 \bar{\psi}-2 \bar{\gamma}}}{\bar{W}_{1}^{2}}\left[\kappa_{4}^{2} \mathrm{e}^{2 \bar{\gamma}}\left(\frac{\bar{X}^{2} \bar{P}^{2}}{2 r^{2}}+\frac{1}{8} \mathrm{e}^{-2 \bar{\psi}} \beta\left(\bar{X}^{2}-\eta^{2}\right)^{2} \bar{W}_{1}^{2}\right)\right. \\
& \left.+\frac{\partial_{t} \bar{W}_{1}^{2}-\partial_{r} \bar{W}_{1}^{2}}{\bar{W}_{1}^{2}}+\frac{\partial_{t t} \bar{W}_{1}-\partial_{r r} \bar{W}_{1}}{\bar{W}_{1}}-2 \frac{\partial_{r} \bar{W}_{1}}{\bar{W}_{1} r}\right] h_{11} \\
& +\frac{\mathrm{e}^{2 \bar{\psi}}}{\bar{W}_{1}^{2} r^{2}}\left[2\left(\partial_{r} \bar{\psi}-\partial_{t} \bar{\psi}+\frac{\partial_{t} \bar{W}_{1}-\partial_{r} \bar{W}_{1}}{\bar{W}_{1}}\right) \partial_{\varphi} h_{14}+2 \frac{\mathrm{e}^{2 \bar{\psi}}}{\bar{W}_{1}^{2} r^{2}}\left(\partial_{r} h_{44}-\partial_{t} h_{44}\right) \dot{h}_{44}\right. \\
& \left.+\partial_{r \varphi} h_{14}-\partial_{t \varphi} h_{14}+\partial_{\varphi \varphi} h_{11}+\frac{\mathrm{e}^{2 \bar{\gamma}}}{r^{2}} \partial_{\varphi \varphi} h_{44}\right]-2 \kappa_{4}^{2} \frac{\mathrm{e}^{2 \bar{\gamma}}}{r^{2}} \epsilon \bar{X}^{2} \bar{P} B
\end{aligned}
$$


and

$$
\begin{aligned}
& \partial_{t} \dot{k}_{14}=\partial_{r} \dot{k}_{14}+2\left(\partial_{r} \bar{\phi}-\partial_{t} \bar{\phi}+\frac{\partial_{t} \bar{W}_{1}-\partial_{r} \bar{W}_{1}}{\bar{W}_{1}}-\frac{1}{r}\right) \dot{k}_{14}+\frac{1}{2} \dot{h}_{55}\left(\partial_{r} h_{14}-\partial_{t} h_{14}\right) \\
& +h_{14}\left(\partial_{r} \dot{h}_{55}-\partial_{t} \dot{h}_{55}\right)+2 \kappa_{4}^{2} \mathrm{e}^{2 \bar{\gamma}-2 \bar{\psi}}\left[\overline { W } _ { 1 } ^ { 2 } \left(\partial_{t} \bar{X} Y\left(n_{1}-n_{2}-\bar{P}\right)\right.\right. \\
& \left.+\bar{X}\left(\bar{P} \partial_{t} Y+e \dot{Y} B\right)\right) \sin \left[\left(n_{2}-n_{1}\right) \varphi\right]+\bar{W}_{1}^{2} \overline{X P} \dot{Z} \sin \left[\left(n_{3}-n_{1}\right) \varphi\right] \\
& \left.+\left(\partial_{t} \bar{X}-\partial_{r} \bar{X}\right) \dot{Y} \mathrm{e}^{2 \bar{\psi}-2 \bar{\gamma}} h_{14} \cos \left[\left(n_{2}-n_{1}\right) \varphi\right]\right] \\
& +\frac{1}{2} \dot{h}_{55}\left(\partial_{\varphi} h_{11}-\bar{W}_{1}^{2} \mathrm{e}^{2 \bar{\gamma}-2 \bar{\psi}} \partial_{\varphi} h_{55}\right)-\frac{\mathrm{e}^{2 \bar{\psi}+2 \bar{\gamma}}}{\bar{W}_{1}^{2} r^{4}} h_{44} \partial_{\varphi} \dot{h}_{44} \\
& -\frac{\mathrm{e}^{2 \bar{\psi}-2 \bar{\gamma}}}{\bar{W}_{1}^{2}}\left(h_{11} \partial_{\varphi} \dot{h}_{11}+\dot{h}_{11} \partial_{\varphi} h_{11}\right)+\partial_{\varphi}\left[\mathrm{e}^{2 \bar{\gamma}-2 \bar{\psi}}\left(\bar{W}_{1}^{2} \partial_{t} \bar{\psi}-\bar{W}_{1} \partial_{t} \bar{W}_{1}\right) h_{55}\right. \\
& +2 h_{11}\left(\partial_{t} \bar{\gamma}+2 \frac{\partial_{t} \bar{W}_{1}}{\bar{W}_{1}}-\partial_{t} \bar{\psi}\right)-\partial_{t} h_{11}+\frac{1}{2}\left(\dot{k}_{22}-\dot{k}_{11}-2 \frac{\mathrm{e}^{2 \bar{\gamma}}}{r^{2}} \dot{k}_{44}\right)+\mathrm{e}^{2 \bar{\gamma}-2 \bar{\psi}} \bar{W}_{1}^{2} \dot{k}_{55} \\
& \left.+2 \frac{\mathrm{e}^{2 \bar{\gamma}}}{r^{2}}\left(\frac{\partial_{t} \bar{W}_{1}}{\bar{W}_{1}}-2 \partial_{t} \bar{\psi}\right) h_{44}-\frac{\mathrm{e}^{2 \bar{\gamma}}}{r^{2}}\left(\partial_{t} h_{44}-\mathrm{e}^{-2 \bar{\psi}} r^{2} \bar{W}_{1}^{2} \partial_{t} h_{55}\right)\right] \\
& +\frac{\mathrm{e}^{2 \bar{\gamma}}}{r^{2}}\left(h_{44} \partial_{\varphi} \dot{h}_{55}-\frac{1}{2} \dot{h}_{44} \partial_{\varphi} h_{55}\right)+2 \kappa_{4}^{2} \bar{W}_{1}^{2} \mathrm{e}^{2 \bar{\gamma}-2 \bar{\psi}}\left(\bar{X}^{2} \bar{P} \epsilon B_{0}-\frac{1}{8} \beta h_{14}\left(\bar{X}^{2}-\eta^{2}\right)^{2}\right)+\mathcal{H},
\end{aligned}
$$

with $\mathcal{H}$ a function of the background fields and the fields $h_{i j}$. The other components of the metric perturbations are obtained in the same way. The second order equations for $\dot{Z}$ and $\dot{C}$ are

$$
\begin{aligned}
& \partial_{t} \dot{Z}=\partial_{r} \dot{Z}+\left(\frac{\partial_{r} \bar{W}_{1}-\partial_{t} \bar{W}_{1}}{\bar{W}_{1}}+\frac{1}{2 r}\right) \dot{Z}-\frac{1}{2} \beta \bar{W}_{1}^{2} \bar{X}^{2} Y \mathrm{e}^{2 \bar{\gamma}-2 \bar{\psi}} \cos \left[\left(n_{3}+n_{2}-2 n_{1}\right) \varphi\right] \\
& +\left[\partial_{r r} Y-\partial_{t t} Y+\frac{\partial_{r} Y}{r}+2 \frac{\partial_{r} Y \partial_{r} \bar{W}_{1}-\partial_{t} Y \partial_{t} \bar{W}_{1}}{\bar{W}_{1}}+\frac{\mathrm{e}^{2 \bar{\psi}-2 \bar{\gamma}}}{\bar{W}_{1}^{2}}\left(\left(\partial_{r} Y-\partial_{t} Y\right) \dot{h}_{11}\right.\right. \\
& \left.+\dot{Y}\left(\partial_{t} h_{11}-\partial_{r} h_{11}\right)\right)-\frac{\mathrm{e}^{2 \bar{\gamma}}}{r^{2}} Y\left(n_{2}-n_{1}+\bar{P}\right)^{2}-\beta \mathrm{e}^{2 \bar{\gamma}-2 \bar{\psi}} Y \bar{X}^{2} \bar{W}_{1}^{2} \\
& +2 \frac{\mathrm{e}^{2 \bar{\psi}}}{\bar{W}_{1}^{2} r^{2}} h_{44} \dot{Y}\left(\partial_{r} \bar{\psi}-\partial_{t} \bar{\psi}-\frac{1}{2 r}\right)+2 \frac{\mathrm{e}^{2 \bar{\psi}-2 \bar{\gamma}}}{\bar{W}_{1}^{2}} h_{11} \dot{Y}\left(\partial_{r} \bar{\gamma}-\partial_{t} \bar{\gamma}-\partial_{r} \bar{\psi}+\partial_{t} \bar{\psi}\right. \\
& \left.\left.+\frac{\partial_{r} \bar{W}_{1}-\partial_{t} \bar{W}_{1}}{\bar{W}_{1}}+\frac{\partial_{r} \dot{Y}-\partial_{t} \dot{Y}}{\dot{Y}}\right)\right] \cos \left[\left(n_{3}-n_{2}\right) \varphi\right] \\
& +\left[\frac { \mathrm { e } ^ { 2 \overline { \psi } - 2 \overline { \gamma } } } { \overline { W } _ { 1 } ^ { 2 } } \left(\frac{1}{2}\left(\partial_{t} \bar{X}-\partial_{r} \bar{X}\right)\left(\dot{k}_{11}-\dot{k}_{22}\right)+\left(\partial_{t} \bar{X} \partial_{t} h_{11}-\partial_{r} \bar{X} \partial_{r} h_{11}\right)\right.\right. \\
& +h_{11}\left(\partial_{r r} \bar{X}-\partial_{t t} \bar{X}+2 \frac{\partial_{r} \bar{X} \partial_{r} \bar{W}_{1}-\partial_{t} \bar{X} \partial_{t} \bar{W}_{1}}{\bar{W}_{1}}\right. \\
& \left.+2\left(\partial_{t} \bar{X} \partial_{t} \bar{\psi}-\partial_{r} \bar{X} \partial_{t} \bar{\psi}+\partial_{r} \bar{X} \partial_{r} \bar{\gamma}-\partial_{t} \bar{X} \partial_{t} \bar{\gamma}\right)\right)+\frac{2 \mathrm{e}^{2 \bar{\psi}} h_{44}}{\bar{W}_{1}^{2} r^{2}}\left(\partial_{r} \bar{X} \partial_{r} \bar{\psi}\right. \\
& \left.\left.-\partial_{t} \bar{X} \partial_{t} \bar{\psi}-\frac{\partial_{r} \bar{X}}{2 r}+\frac{\mathrm{e}^{-2 \bar{\gamma}} \overline{X P}^{2}}{2 r^{2}}\right)-\frac{2 \epsilon \mathrm{e}^{2 \bar{\gamma}}}{r^{2}} \bar{X} \bar{P} B\right] \cos \left[\left(n_{3}-n_{1}\right) \varphi\right],
\end{aligned}
$$




$$
\begin{aligned}
\partial_{t} \dot{C} & =\partial_{r} \dot{C}+\left(\partial_{r} \bar{\psi}-\partial_{t} \bar{\psi}-\frac{1}{2 r}\right) \dot{C}+\frac{1}{2} \mathrm{e}^{2 \bar{\gamma}-2 \bar{\psi}} \epsilon^{2} \bar{X}^{2} \bar{W}_{1}^{2} B \\
& +\frac{1}{2}\left(\partial_{r r} B-\partial_{t t} B+\partial_{t \varphi} B_{0}-\partial_{r \varphi} B_{0}\right)+\frac{1}{2} \mathrm{e}^{2 \bar{\gamma}-2 \bar{\psi}} \epsilon \bar{X} Y \bar{W}_{1}^{2}\left(n_{2}-n_{1}+2 \bar{P}\right) \cos \left[\left(n_{2}-n_{1}\right) \varphi\right] \\
& +\frac{\mathrm{e}^{2 \bar{\psi}-2 \bar{\gamma}}}{\bar{W}_{1}^{2}}\left[\partial_{t} \dot{B}-\partial_{r} \dot{B}+\frac{1}{2 \epsilon}\left(\partial_{t t} \bar{P}-\partial_{r r} \bar{P}+\frac{\partial_{r} \bar{P}}{r}\right)+\dot{B}\left(\frac{\partial_{r} \bar{W}_{1}-\partial_{t} \bar{W}_{1}}{\bar{W}_{1}}+\frac{1}{2 r}\right)\right. \\
& \left.+\frac{\partial_{r} \bar{P} \partial_{r} \bar{W}_{1}-\partial_{t} \bar{P} \partial_{t} \bar{W}_{1}}{\epsilon \bar{W}_{1}}\right] h_{11}+\frac{\mathrm{e}^{2 \bar{\psi}}}{\bar{W}_{1}^{2} r^{2} \epsilon}\left[2\left(\partial_{t} \bar{P} \partial_{t} \bar{\psi}-\partial_{r} \bar{P} \partial_{r} \bar{\psi}\right)\right. \\
& \left.+2 \dot{B} \epsilon\left(\partial_{t} \bar{\psi}-\partial_{r} \bar{\psi}+\frac{1}{2 r}\right)+\frac{\partial_{r} \bar{P}}{r}+\frac{\mathrm{e}^{2 \bar{\psi}}}{2 \bar{W}_{1}^{2} r^{2}}\left(\partial_{r} \bar{P}-\partial_{t} \bar{P}\right) \dot{h}_{44}\right] h_{44} \\
& +\frac{\mathrm{e}^{2 \bar{\psi}}}{2 \bar{W}_{1}^{2} r^{2} \epsilon}\left(\left(\partial_{t} \bar{P}-\partial_{r} \bar{P}\right) \dot{k}_{44}+\partial_{t} \bar{P} \partial_{t} h_{44}-\partial_{r} \bar{P} \partial_{r} h_{44}+\epsilon \dot{B}\left(\partial_{t} h_{44}-\partial_{r} h_{44}\right)\right) .
\end{aligned}
$$

There are the second order partial derivative terms $\partial_{t t} Y-\partial_{r r} Y$ and $\partial_{t t} B-\partial_{r r} B$ in Equation (35) and Equation (36) respectively. They can be isolated in order to get a wave equation for the first order perturbations.

\section{The Energy Momentum Components}

For the several orders of the energy-momentum tensor components we find

$$
\begin{aligned}
& { }^{4} \bar{T}_{t \varphi}=0, \\
& { }^{4} T_{t \varphi}^{(0)}=\overline{X P} \dot{Y} \sin \left[\left(n_{2}-n_{1}\right) \varphi\right] \\
& { }^{4} T_{t \varphi}^{(1)}=\left[\partial_{t} \bar{X} Y\left(n_{1}-n_{2}-\bar{P}\right)+\bar{X}\left(\bar{P} \partial_{t} Y+\epsilon B \dot{Y}\right)\right] \sin \left[\left(n_{2}-n_{1}\right) \varphi\right] \\
& +\overline{X P} \dot{Z} \sin \left[\left(n_{3}-n_{1}\right) \varphi\right]+\frac{\mathrm{e}^{2 \bar{\psi}-2 \bar{\gamma}}}{\bar{W}_{1}^{2}} \dot{Y} h_{14}\left(\partial_{t} \bar{X}-\partial_{r} \bar{X}\right) \cos \left[\left(n_{2}-n_{1}\right) \varphi\right] \\
& +\bar{X}^{2} \bar{P} \epsilon B_{0}-\frac{1}{8} \beta\left(\bar{X}^{2}-\eta^{2}\right)^{2} h_{14}-\frac{\mathrm{e}^{2 \bar{\psi}-2 \bar{\gamma}}}{2 \bar{W}_{1}^{2}} h_{14}\left[\frac{\mathrm{e}^{2 \bar{\psi}}}{r^{2} \bar{W}_{1}^{2} \epsilon^{2}}\left(\partial_{t} \bar{P}-\partial_{r} \bar{P}\right)^{2}+\partial_{r} \bar{X}^{2}-\partial_{t} \bar{X}^{2}+\mathrm{e}^{2 \bar{\gamma}} \frac{\bar{X}^{2} \bar{P}^{2}}{r^{2}}\right] . \\
& { }^{4} \bar{T}_{t t}=\frac{\mathrm{e}^{2 \bar{\psi}}}{2 \bar{W}_{1}^{2} r^{2} \epsilon^{2}}\left(\partial_{r} \bar{P}^{2}+\partial_{t} \bar{P}^{2}\right)+\frac{1}{2}\left(\partial_{t} \bar{X}^{2}+\partial_{r} \bar{X}^{2}\right)+\frac{1}{2 r^{2}} \mathrm{e}^{2 \bar{\gamma}} \bar{X}^{2} \bar{P}^{2}+\frac{1}{8} \mathrm{e}^{2 \bar{\gamma}-2 \bar{\psi}} \bar{W}_{1}^{2} \beta\left(\bar{X}^{2}-\eta^{2}\right)^{2} \\
& { }^{4} T_{t t}^{(0)}=\dot{Y}^{2}+\dot{Y}\left(\partial_{t} \bar{X}+\partial_{r} \bar{X}\right) \cos \left[\left(n_{2}-n_{1}\right) \varphi\right]+\frac{\mathrm{e}^{2 \bar{\psi}}}{\bar{W}_{1}^{2} r^{2} \epsilon}\left(\epsilon \dot{B}^{2}+\dot{B}\left(\partial_{r} \bar{P}+\partial_{t} \bar{P}\right)\right) \\
& { }^{4} T_{t t}^{(1)}=\dot{Z}\left(\partial_{t} \bar{X}+\partial_{r} \bar{X}\right) \cos \left[\left(n_{3}-n_{1}\right) \varphi\right]+2 \dot{Y} \dot{Z} \cos \left[\left(n_{3}-n_{2}\right) \varphi\right] \\
& +2 \epsilon \bar{X} \dot{Y} B_{0} \sin \left[\left(n_{2}-n_{1}\right) \varphi\right]+\bar{X} Y\left(\frac{\beta}{2} \mathrm{e}^{2 \bar{\gamma}-2 \bar{\psi}} \bar{W}_{1}^{2}\left(\bar{X}^{2}-\eta^{2}\right)\right. \\
& \left.+\frac{\partial_{r} \bar{X} \partial_{r} Y+\partial_{t} \bar{X} \partial_{t} Y}{\bar{X} Y}+\frac{\mathrm{e}^{2 \bar{\gamma}}}{r^{2}} Y\left(n_{2}-n_{1}+\bar{P}\right)\right) \cos \left[\left(n_{2}-n_{1}\right) \varphi\right] \\
& -\frac{\mathrm{e}^{4 \bar{\psi}}}{\bar{W}_{1}^{4} r^{4} \epsilon^{2}}\left(\frac{1}{2}\left(\partial_{t} \bar{P}^{2}+\partial_{r} \bar{P}^{2}\right)+\epsilon \dot{B}\left(\partial_{t} \bar{P}+\partial_{r} \bar{P}\right)+\epsilon^{2} \dot{B}^{2}\right. \\
& \left.+\frac{1}{2} \mathrm{e}^{2 \bar{\gamma}-2 \bar{\psi}} \bar{X}^{2} \bar{P}^{2} \bar{W}_{1}^{2} \epsilon^{2}\right) h_{44}-\left(\frac{1}{8} \beta\left(\bar{X}^{2}-\eta^{2}\right)^{2}+\frac{\mathrm{e}^{2 \bar{\psi}}}{2 \bar{W}_{1}^{2} r^{2}} \bar{X}^{2} \bar{P}^{2}\right) h_{11} \\
& +\dot{Y}\left(\partial_{t} Y+\partial_{r} Y\right)+\frac{\mathrm{e}^{2 \bar{\psi}}}{\bar{W}_{1}^{2} r^{2} \epsilon} \dot{C}\left(\partial_{t} \bar{P}+\partial_{r} \bar{P}+2 \epsilon \dot{B}\right)+\frac{\epsilon \mathrm{e}^{2 \bar{\gamma}}}{r^{2}} \bar{X}^{2} \bar{P}^{2} B
\end{aligned}
$$




$$
\begin{aligned}
& { }^{4} \bar{T}_{\varphi \varphi}=\frac{\mathrm{e}^{2 \bar{\psi}-2 \bar{\gamma}}}{2 \bar{W}_{1}^{2} \epsilon^{2}}\left(\partial_{r} \bar{P}^{2}-\partial_{t} \bar{P}^{2}\right)+\frac{1}{2} \mathrm{e}^{-2 \bar{\gamma}} r^{2}\left(\partial_{t} \bar{X}^{2}-\partial_{r} \bar{X}^{2}\right) \\
& +\frac{1}{2} \bar{X}^{2} \bar{P}^{2}-\frac{1}{8} \mathrm{e}^{-2 \bar{\psi}} \bar{W}_{1}^{2} r^{2} \beta\left(\bar{X}^{2}-\eta^{2}\right)^{2} \\
& { }^{4} T_{\varphi \varphi}^{(0)}=\mathrm{e}^{-2 \bar{\gamma}} r^{2} \dot{Y}\left(\partial_{t} \bar{X}-\partial_{r} \bar{X}\right) \cos \left[\left(n_{2}-n_{1}\right) \varphi\right]+\mathrm{e}^{2 \bar{\psi}-2 \bar{\gamma}} \frac{\dot{B}}{\bar{W}_{1}^{2} \epsilon}\left(\partial_{r} \bar{P}-\partial_{t} \bar{P}\right) \\
& { }^{4} T_{\varphi \varphi}^{(1)}=\left[\frac{\mathrm{e}^{-2 \bar{\psi}}}{2} \bar{W}_{1}^{2} r^{2} Y \bar{X} \beta\left(\eta^{2}-\bar{X}^{2}\right)+\mathrm{e}^{-2 \bar{\gamma}} r^{2}\left(\partial_{t} \bar{X} \partial_{t} Y-\partial_{r} \bar{X} \partial_{r} Y\right)\right. \\
& +\overline{X P} Y\left(n_{2}-n_{1}+\bar{P}\right)+\frac{\mathrm{e}^{2 \bar{\psi}-2 \bar{\gamma}}}{\bar{W}_{1}^{2}} \dot{Y}\left(\partial_{t} \bar{X}-\partial_{r} \bar{X}\right)\left(\left(h_{44}+r^{2} \mathrm{e}^{-2 \bar{\gamma}} h_{11}\right)\right] \cos \left[\left(n_{2}-n_{1}\right) \varphi\right] \\
& +\left(r^{2} \mathrm{e}^{-2 \bar{\gamma}} \dot{Z}\left(\partial_{t} \bar{X}-\partial_{r} \bar{X}\right)\right) \cos \left[\left(n_{3}-n_{1}\right) \varphi\right]-\frac{1}{8} \beta h_{44}\left(\bar{X}^{2}-\eta^{2}\right)^{2} \\
& +r^{2} \mathrm{e}^{-2 \bar{\gamma}} \dot{Y}\left(\partial_{t} Y-\partial_{r} Y\right)+\frac{\mathrm{e}^{2 \bar{\psi}-2 \bar{\gamma}}}{\bar{W}_{1}^{2} \epsilon} \dot{C}\left(\partial_{r} \bar{P}-\partial_{t} \bar{P}\right)+\frac{\overline{X P} \epsilon \dot{B}}{r^{2}} \\
& +\frac{\mathrm{e}^{2 \bar{\psi}-4 \bar{\gamma}}}{2 \bar{W}_{1}^{2}}\left(r^{2} h_{11}+\mathrm{e}^{2 \bar{\gamma}} h_{44}\right)+\frac{\mathrm{e}^{4 \bar{\psi}-4 \bar{\gamma}}}{2 \bar{W}_{1}^{4} \epsilon^{2}} h_{11}\left(\partial_{r} \bar{P}^{2}-\partial_{t} \bar{P}^{2}+\epsilon \dot{B}\left(\partial_{r} \bar{P}-\partial_{t} \bar{P}\right)\right) . \\
& { }^{4} T_{z z}^{(0)}=\mathrm{e}^{4 \bar{\psi}-2 \bar{\gamma}} \dot{Y}\left(\partial_{t} \bar{X}-\partial_{r} \bar{X}\right) \cos \left[\left(n_{2}-n_{1}\right) \varphi\right]+\frac{\mathrm{e}^{6 \bar{\psi}-2 \bar{\gamma}}}{\bar{W}_{1}^{2} r^{2} \epsilon} \dot{B}\left(\partial_{t} \bar{P}-\partial_{r} \bar{P}\right) .
\end{aligned}
$$

Submit or recommend next manuscript to SCIRP and we will provide best service for you:

Accepting pre-submission inquiries through Email, Facebook, LinkedIn, Twitter, etc. A wide selection of journals (inclusive of 9 subjects, more than 200 journals)

Providing 24-hour high-quality service

User-friendly online submission system

Fair and swift peer-review system

Efficient typesetting and proofreading procedure

Display of the result of downloads and visits, as well as the number of cited articles Maximum dissemination of your research work

Submit your manuscript at: http://papersubmission.scirp.org/

Or contact jmp@scirp.org 